08

\title{
Формирование неклассических многофотонных состояний света со сжатыми квантовыми флуктуациями в волокнах из модифицированного висмутом теллуритного стекла
}

\author{
(C) A.A. Сорокин ${ }^{1}$, В.В. Дорофреев ${ }^{1,2}$, С.Е. Моторин ${ }^{1,2}$, I. Lyashuk $^{3}$, J. Porins ${ }^{3}$, G. Leuchs ${ }^{1,4}$, V. Bobrovs $^{3}$ \\ ${ }^{1}$ Федеральный исследовательский центр Институт прикладной физики РАН, \\ 603950 Нижний Новгород, Россия \\ ${ }^{2}$ Институт химии высокочистых веществ им. Г.Г. Девятых РАН, \\ 603950 Нижний Новгород, Россия \\ ${ }^{3}$ Riga Technical University, Institute of Telecommunications, \\ 1048 Riga, Latvia \\ ${ }^{4}$ Max Planck Institute for the Science of Light, \\ D-91058 Erlangen, Germany \\ e-mail: arsorok1997@yandex.ru
}

Поступила в редакцию 20.12.2021 г.

В окончательной редакции 20.12.2021 г.

Принята к публикации 30.12.2021 г.

\begin{abstract}
Предложено и изготовлено оптическое волокно с высоким значением коэффициента керровской нелинейности из модифицированного висмутом теллуритного стекла для формирования неклассических многофотонных состояний света. А именно предложено использовать данное волокно для сжатия квантовых флуктуаций одной из квадратурных компонент оптического сигнала мощностью $20 \mathrm{~W}$ до уровня значительно сильнее $-10 \mathrm{~dB}$ относительно стандартного квантового предела, что может быть важно для различных приложений. С помощью численного моделирования в рамках стохастического нелинейного уравнения Шредингера было продемонстрировано сжатие шумов лучше, чем $-16 \mathrm{~dB}$, при оптимальных длинах теллуритного волокна 6-14 m, в то время как в кварцевых волокнах ожидается сжатие $-14 \mathrm{~dB}$ при использовании значительно более длинных отрезков волокна $120-300 \mathrm{~m}$. Для установления физических эффектов, ограничивающих сжатие, выполнены оценки с помощью аналитических формул.
\end{abstract}

Ключевые слова: сжатие квантовых флюктуаций, керровская нелинейность, оптические волокна, стохастическое нелинейное уравнение Шредингера, модифицированные висмутом теллуритные стекла.

DOI: $10.21883 /$ OS.2022.04.52270.54-21

\section{Введение}

Развитие квантовых технологий позволяет получать неклассические состояния света с уникальными характеристиками, что способствует развитию новых приложений и глубокому пониманию фундаментальных основ физики [1-3]. Зачастую под квантовыми состояниями света понимаются состояния с малым числом фотонов, однако и для многофотонных состояний статистика может сильно отличаться от классической [3]. Одним из наиболее значимых применений многофотонных неклассических состояний света является детектирование гравитационных волн. Первое прямое обнаружение гравитационных волн в лазерно-интерферометрической гравитационно-волновой обсерватории aLIGO (advanced Laser Interferometer Gravitational-Wave Observatory) стало началом эры астрономии гравитационных волн [4]. Увеличение частоты регистрируемых событий и отношения сигнал/шум требует повышения чувствительности детекторов, которая обычно ограничивается классическими и квантовыми шумами. Для достижения проектной чувствительности в детекторах гравитационных волн нового поколения требуется сжатие широкопо- лосного квантового шума одной из квадратур сигнала до уровня $-10 \mathrm{~dB}$ относительно квантового предела [5] (при этом дисперсия флуктуаций квадратуры, сопряженной сжатой, увеличивается для выполнения принципа неопределенности Гейзенберга [6]). Помимо задач детектирования гравитационных волн сжатый свет также востребован для прецизионной метрологии, квантовых коммуникаций и др. [2,6-9]. Поэтому разработка источников неклассического многофотонного света со сжатыми квантовыми флуктуациями является востребованной задачей. В настоящее время источники мощностью от нескольких ватт до сотни ватт с уровнем шумов, практически равным стандартному квантовому пределу, продемонстрированы различными научными группами [1012]. Для дальнейшего сжатия шума таких источников могут применяться различные методы, основанные на нелинейно-оптических эффектах, что подробно описано в обзорной статье [6]. Один из методов основан на использовании керровской нелинейности оптических волокон. В этом случае уменьшение дисперсии флуктуаций происходит для квадратуры, повернутой под некоторым оптимальным углом в фазовом пространстве 


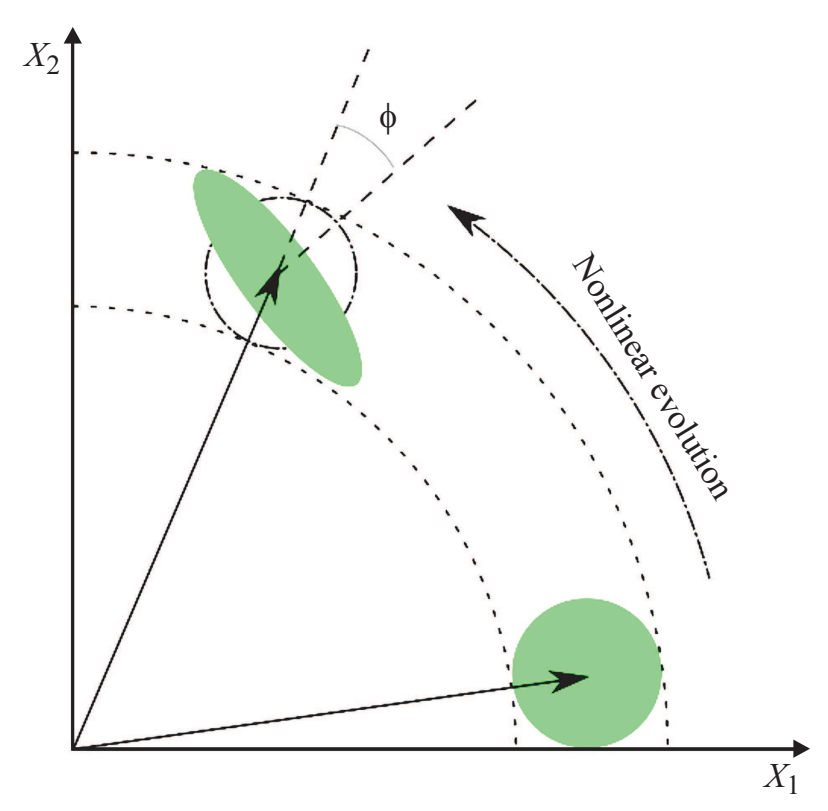

Рис. 1. Иллюстрация сжатия флуктуаций в среде с керровской нелинейностью. Круг внизу - квантовые шумы для когерентного состояния на фазовой плоскости квадратур $X_{1}$ и $X_{2}$, преобразующиеся в эллипс в процессе нелинейной эволюции сигнала. Для этого эллипса флуктуации повернутой на угол $\phi$ квадратуры меньше флуктуаций для когерентного состояния, поэтому такое состояние называется сжатым.

(рис. 1) [6]. Для сжатия квантовых шумов света в волокнах с керровской нелинейностью обычно используются кварцевые световоды: сплошные $[13,14]$ или фотоннокристаллические $[15,16]$. Недавно было предложено и теоретически исследовано формирование сжатого света в специальных световодах с огромной керровской нелинейностью из халькогенидных стекол [17]. В настоящей работе мы исследуем сжатие квантовых шумов в специально разработанных и созданных нами волокнах из модифицированных висмутом теллуритных стекол с высокой нелинейностью.

Известно, что теллуритные волоконные световоды со специально разработанными конструкциями представляют огромный интерес для нелинейно-оптических преобразований когерентного излучения благодаря высоким значениям кубичных нелинейных откликов и широкой полосе прозрачности. Так, в теллуритных волокнах продемонстрирована генерация спектрального суперконтинуума с шириной более двух октав $[18,19]$, исследовано получение частотно-перестраиваемых солитонов [20-23], изучены процессы четырехволнового взаимодействия [24,25]. Также показана возможность применения многосердцевинных теллуритных волокон с управляемой дисперсией [26] для широкополосного преобразования ультракоротких импульсов со специальным пространственным распределением фазы [27]. Активные элементы на основе теллуритных световодов, легированных редкоземельными ионами, имеют огром- ные перспективы для генерации лазерного излучения с управляемыми параметрами [28-33]. Возможности применения теллуритных волокон для реализации квантовых эффектов ранее практически не исследовались. Однако благодаря высоким значениям керровской нелинейности по сравнению со стандартными кварцевыми волокнами для получения одинакового набега нелинейной фазы, определяющего сжатие флуктуаций, длины теллуритных волокон должны быть значительно меньше, чем длины кварцевых волокон. Это является огромным преимуществом, так как позволяет подавить в теллуритных волокнах вынужденное рассеяние МандельштамаБриллюэна на звуковых волнах (GAWBS - guided acoustic wave Brillouin scattering), пропорциональное длине волокна [34].

В настоящей работе мы изготовили волокно из модифицированного висмутом теллуритного стекла и провели теоретический анализ формирования неклассических многофотонных состояний света со сжатыми квантовыми флуктуациями в данном волокне в сравнении со стандартным кварцевым волокном.

\section{Изготовление волокна из модифицированного висмутом теллуритного стекла}

Мы изготовили одномодовый волоконный световод с сердцевиной и двумя оболочками диаметрами $12 / 100 / 360 \mu \mathrm{m}$ из стекол цинк-теллуритной системы. Полученное с помощью оптического микроскопа изображение торца изготовленного волокна приведено на рис. 2. Выбор цинк-теллуритной системы обусловлен тем, что некоторые составы стекол из этой группы на основе диоксида теллура $\mathrm{TeO}_{2}$ обладают высокой устойчивостью против кристаллизации и малым поглощением в инфракрасном диапазоне [35-37], позволяя изготавливать на их основе световоды высокого качества с низкими потерями $(20 \mathrm{~dB} / \mathrm{km}$ [38]). Увеличение показателей преломления сердцевины относительно первой оболочки и первой оболочки относительно второй оболочки достигалось путем добавлением оксида висмута $\mathrm{Bi}_{2} \mathrm{O}_{3}$ в состав стекла. Измеренные показатели преломления стекол сердцевины и первой оболочки составили $n_{\text {core }}=2.0236$ и $n_{\text {clad } 1}=2.02$ соответственно, что дает значение числовой апертуры $N A=0.12$. Для изготовленного волокна рассчитывались коэффициенты дисперсии групповых скоростей $\beta_{2}$ и керровской нелинейности $\gamma$ с помощью конечно-элементного метода (как и в работе [26]). Полученные значения $\beta_{2}=150 \mathrm{ps}^{2} / \mathrm{km}$ и $\gamma=30(\mathrm{~W} \mathrm{~km})^{-1}$ применялись при моделировании нелинейной эволюции оптических сигналов в данном волокне.

Процедура синтеза теллуритных стекол, похожих на применяемые в настоящей работе стекла для изготовления волоконного световода, более подробно описана в [35,39]. Синтез стекол осуществлялся с помощью 


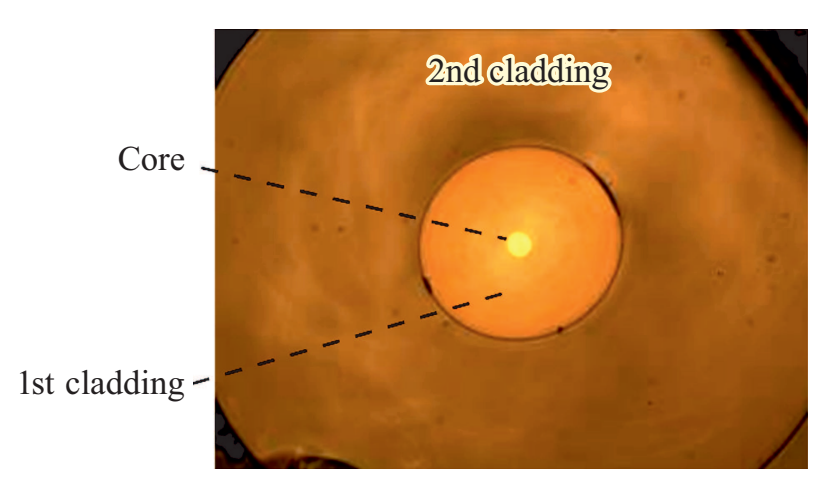

Pис. 2. Полученное на оптическом микроскопе изображение торца волокна из теллуритного стекла, модифицированного висмутом.

специальной установки путем плавления оксидов в платиновом тигле в атмосфере $\mathrm{O}_{2}$. В качестве исходных высокочистых компонентов применялись полученные по оригинальным технологиям $\mathrm{TeO}_{2}$ и $\mathrm{ZnO}$. В качестве модифицирующих добавок применялись коммерчески доступные „ОСЧ“-оксиды. Синтезированные стекла содержат менее $0.1-2$ ppmwt $3 d$-переходных металлов в сумме и менее 1-2 ppmwt ионов редкоземельных металлов (для каждого вида иона), что ниже предела обнаружения лазерной масс-спектроскопии $[35,40]$. Из стекол для сердцевины и 1-й оболочки изготавливалась монолитная двухслойная преформа, а из стекла для 2-й оболочки изготавливалась жакетирующая трубка. Данная технология изготовления волоконных световодов со „степ-индексом“ из монолитной заготовки позволяет защитить оболочкой высокочистое стекло сердцевины от внешних загрязнений на всех стадиях технологических процессов, что позволяет получать образцы с низкими оптическими потерями. Волоконный световод изготавливался путем вытяжки из сборки, составленной из жакетирующей трубки + растянутой в палочку преформы. Границы раздела между сердцевиной и 1-й оболочкой, а также между 1-й и 2-й оболочками хорошо заметны и не содержат видимых дефектов, как видно на рис. 2.

\section{Теоретический анализ сжатия квантовых флуктуаций оптических сигналов}

Теоретический анализ формирования неклассических многофотонных состояний света со сжатыми квантовыми флуктуациями основан на численном моделировании нелинейной эволюции непрерывных оптических сигналов с учетом квантовых шумов в теллуритном и кварцевом волоконных световодах. Для этого мы использовали модифицированное стохастическое обобщенное нелинейное уравнение Шредингера в представлении
Вигнера $[41,42]$ :

$$
\begin{aligned}
& \frac{\partial A(t, z)}{\partial z}=+\left[i \gamma \int R(t-s)|A(s, z)|^{2} d s+\Gamma^{\mathrm{R}}(t, z)\right] \\
& \times A(t, z)-\alpha A(t, z)+\Gamma(t, z)+i \frac{\beta_{2}}{2} \frac{\partial^{2}}{\partial t^{2}} A(t, z),
\end{aligned}
$$

где $A(t, z)$ - комплексная амплитуда оптического поля, $t$ - время в сопровождающей системе координат, $z-$ координата вдоль оси волокна, $\alpha-$ классические линейные потери, $R(t)$ - детерминированный нелинейный (кубичный) отклик среды, учитывающий мгновенную керровскую и задержанную рамановскую нелинейности, функции Г и $\Gamma^{\mathrm{R}}$ введены для учета линейного квантового шума и рамановского шума соответственно $[41,42]$. Г и $\Gamma^{\mathrm{R}}$ представляют собой $\delta$-коррелированные случайные величины со средним значением, равным 0, и нормальным распределением в спектральной области [41-43]:

$$
\begin{gathered}
\Gamma(\omega, z)=\frac{1}{\sqrt{2 \pi}} \int_{-\infty}^{\infty} \Gamma(t, z) e^{i \omega t} d t, \\
\Gamma^{\mathrm{R}}(\omega, z)=\frac{1}{\sqrt{2 \pi}} \int_{-\infty}^{\infty} \Gamma^{\mathrm{R}}(t, z) e^{i \omega t} d t, \\
\left\langle\Gamma(\omega, z) \Gamma^{*}\left(\omega^{\prime}, z^{\prime}\right)\right\rangle=\alpha \hbar \omega_{0} \delta\left(\omega-\omega^{\prime}\right) \delta\left(z-z^{\prime}\right), \\
\left\langle\Gamma^{\mathrm{R}}(\omega, z) \Gamma^{\mathrm{R}^{*}}\left(\omega^{\prime}, z^{\prime}\right)\right\rangle= \\
=\alpha^{\mathrm{R}}(\omega) \gamma \hbar \omega_{0}\left[\frac{1}{2}+\frac{1}{e^{\frac{\hbar|\omega|}{k T}}-1}\right] \delta\left(\omega \omega^{\prime}\right) \delta\left(z-z^{\prime}\right),
\end{gathered}
$$

$\omega_{0}$ - несущая частота $\left(\omega_{0}=2 \pi c / \lambda_{0}, c-\right.$ скорость света в вакууме, $\lambda_{0}$ - длина волны входного сигнала), $\omega$ - разность рассматриваемой частоты и несущей частоты, $k$ - постоянная Больцмана, $T-$ температура, $\delta(t)$ - дельта-функция Дирака, $\hbar$ - постоянная Планка.

Функция нелинейного отклика $R(t)$ задавалась в виде

$$
R(t)=f_{\mathrm{R}} \delta(t)+\left(1-f_{\mathrm{R}}\right) h(t),
$$

где $f_{\mathrm{R}}$ - доля вклада рамановского эффекта в общую нелинейность, а $h(t)$ - форма рамановской функции. Функция отклика для кварцевого стекла взята из работы [41], а для теллуритного стекла - из [44]. На рис. $3, a, b$ показаны действительные и мнимые части функций отклика в спектральном представлении.

Численное моделирование уравнения (1) проводилось с помощью разработанного нами компьютерного кода, основанного на методе фурье-расщепления по физическим факторам (SSFM, split-step Fourier method) [43]. Использовались циклические граничные условия во временном пространстве. В качестве начальных условий задавалось оптическое поле с постоянными фазой и амплитудой и дополнительным стохастическим шумом $\delta A(t, 0)$ с нормальным распределением:

$$
A(t, 0)=\sqrt{P_{0}}+\delta A(t, 0)
$$



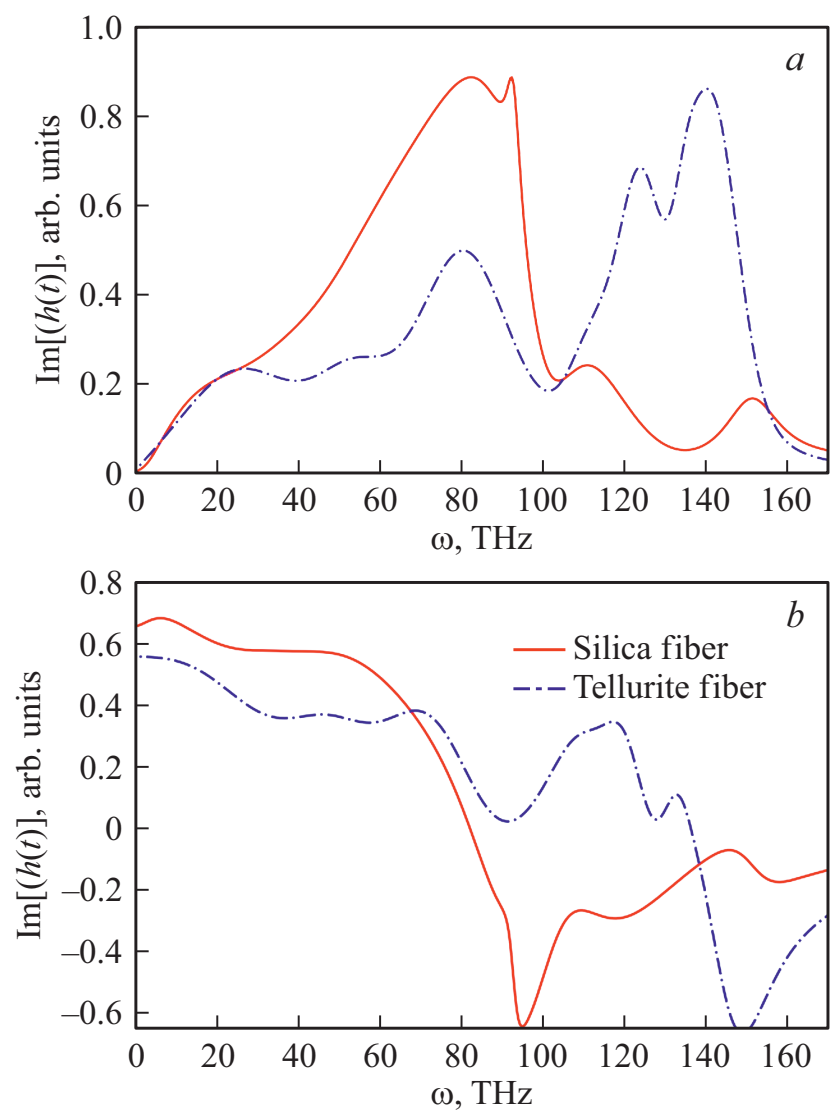

Рис. 3. Действительные и мнимые части функций рамановского отклика в спектральном представлении для теллуритного и кварцевого стекол.

$$
\left\langle\delta A(t, 0) \delta A^{*}(t, 0)\right\rangle=\frac{\hbar \omega_{0}}{2} \delta\left(t-t^{\prime}\right),
$$

где $P_{0}-$ мощность входного оптического сигнала.

Для получения статистики эволюции квантовых флуктуаций многофотонных состояний света при распространении в волокне мы запустили 1000 раз численный код, моделирующий уравнение (1) с учетом формул (2)-(8) с различными реализациями начального шума и источников шума вдоль волокна. По полученным данным эволюции сигналов мы вычислили величину сжатия квантовых шумов, используя методику из [43]. Параметры, использованные при моделировании кварцевого и теллуритного волокон, приведены в таблице.

Для сравнения влияния физических факторов, ограничивающих сжатие флуктуаций в волоконных световодах с керровской нелинейностью были использованы упрощенные аналитические формулы, полученные без учета рамановских эффектов и позволяющие оценить сжатие шумов в волокне, пренебрегая потерями $\left(V_{0}\right)$ и „концентрируя“ все потери в волокне на выходе из него $\left(V_{\text {loss }}\right)[3]$ :

$$
\begin{gathered}
V_{0}=10 \log _{10}\left(1-2 r_{\text {Kerr }} \sqrt{1+r_{\text {Kerr }}^{2}}+2 r_{\text {Kerr }}^{2}\right), \\
V_{\text {loss }}=10 \log _{10}\left[(1-R) 10^{V_{0} / 10}+R\right] .
\end{gathered}
$$

Параметры, использованные при моделировании

\begin{tabular}{c|c|c}
\hline Параметры & Теллуритное волокно & Кварцевое волокно \\
\hline$\beta_{2}, \mathrm{ps}^{2} / \mathrm{km}$ & 150 & -20.0 \\
$\gamma, 1 /(\mathrm{W} \cdot \mathrm{km})$ & 30.0 & 1.0 \\
$\alpha, \mathrm{dB} \cdot \mathrm{km}^{-1}$ & 20.0 & 1.0 \\
$\lambda_{0}, \mu \mathrm{m}$ & 1.55 & 1.55 \\
$T, \mathrm{~K}$ & 300 & 300 \\
$P_{0}, \mathrm{~W}$ & 20.0 & 20.0
\end{tabular}
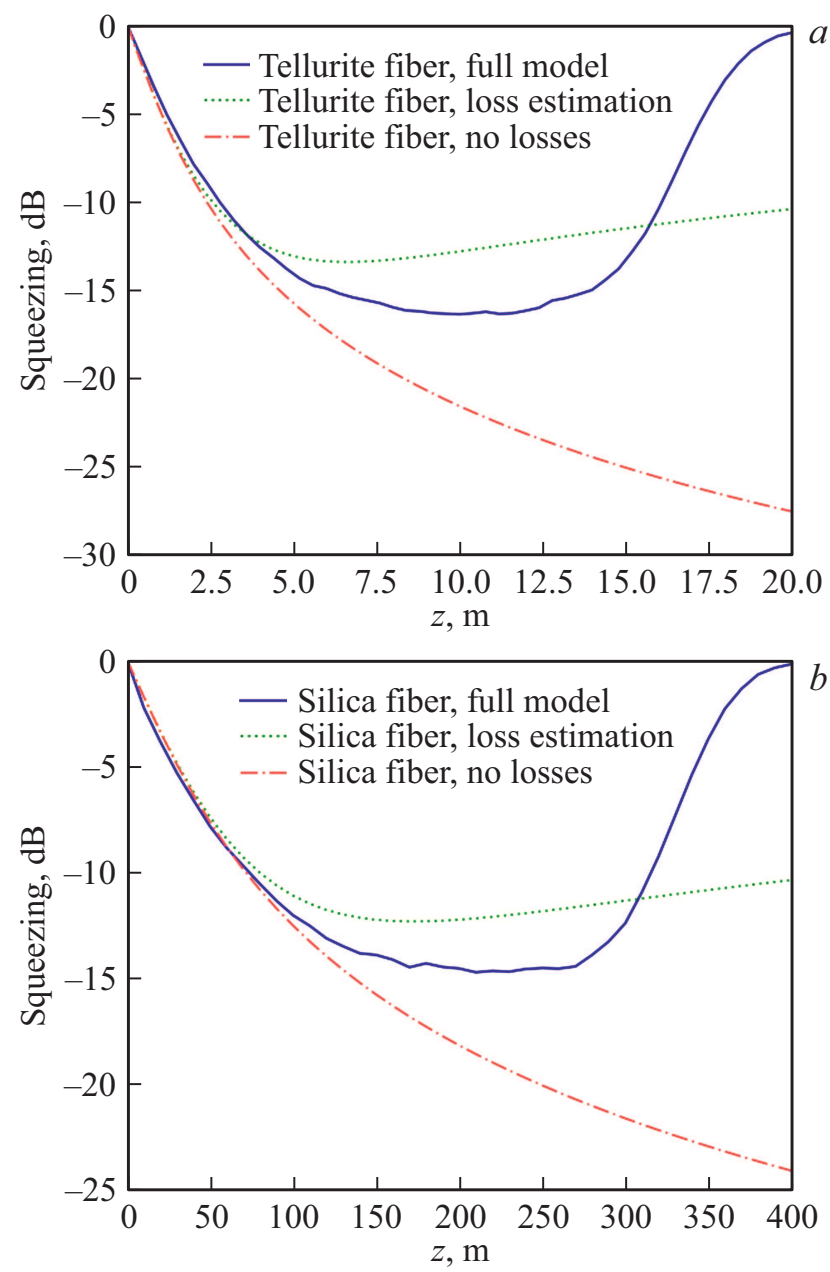

Рис. 4. Результаты моделирования сжатия квантовых флуктуаций для теллуритного и кварцевого волокон.

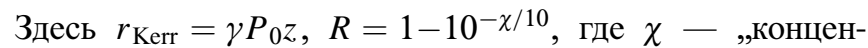

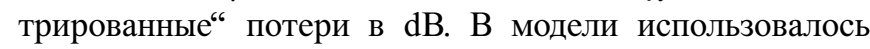
$\chi=\alpha z$.

Результаты численного моделирования представлены на рис. 4. Видно, что в волокнах из теллуритных стекол, модифицированных висмутом, возможно сжатие $-16 \mathrm{~dB}$. В кварцевых волокнах достигается значение $-14 \mathrm{~dB}$, что немного хуже. Но важно отметить, что характерные длины теллуритных световодов для достижения близкого к оптимальному сжатию составляют 6-14 m, а характерные длины кварцевых световодов 
составляют 120-300 m. Напомним, что в численном моделировании не учитывается GAWBS, которое значительно ухудшает сжатие шумов в длинных волокнах $(>100 \mathrm{~m})$ [34], поэтому результаты в эксперименте для кварцевых волокон будут значительно хуже, чем на рис. 4, $b$, в то время как для теллуритных волокон изза их относительно небольших длин $(\sim 10 \mathrm{~m})$ GAWBS практически не скажется.

Также важно подчеркнуть, что в диапазонах оптимальных длин волоконных световодов аналитические приближения перестают точно описывать сжатие и дают лишь оценку сверху (для формулы (9)) и снизу (для формулы (10)). Формула (10) в этом диапазоне оценивает сжатие снизу, поскольку при таком учете влияния потерь не учитывается сжатие шумов, вызванных потерями в начале волокна, на оставшейся его части [43]. На длинах $>14 \mathrm{~m}$ для теллуритных волокон и $>300 \mathrm{~m}$ для кварцевых волокон влияние рамановских эффектов становится преобладающим, поэтому формулы (9) и (10), не учитывающие эти эффекты, перестают описывать поведение силы подавления квантовых флуктуаций в волокне даже качественно.

\section{Заключение}

Предложено и изготовлено оптическое волокно с высоким значением коэффициента керровской нелинейности, $\gamma=30(\mathrm{~W} \cdot \mathrm{km})^{-1}$, из модифицированного висмутом теллуритного стекла для формирования неклассических многофотонных состояний света. А именно предложено использовать данное волокно для сжатия квантовых флуктуаций одной из квадратурных компонент оптического сигнала мощностью $20 \mathrm{~W}$ до уровня значительно сильнее $-10 \mathrm{~dB}$ относительно стандартного квантового предела, что может быть важно для различных приложений, включая детектирование гравитационных волн. Эволюция непрерывного лазерного сигнала с учетом квантовых шумов была численно промоделирована при помощи стохастического нелинейного уравнения Шредингера. Для получения достоверной статистики было выполнено 1000 запусков с различными реализациями начального шума и источников шума вдоль волокна. На основе этих запусков была найдена величина подавления квантовых флуктуаций в волокнах из модифицированного висмутом теллуритного стекла и для сравнения в волокнах из кварцевого стекла. Установлено, что в теллуритном волокне достижимо более сильное сжатие $-16 \mathrm{~dB}$ по сравнению со сжатием $-14 \mathrm{~dB}$ в волокнах из кварцевого стекла без учета рассеяния на акустических волнах. Однако для оптимального сжатия требуемые длины теллуритных волокон 6-14 m на порядок меньше, чем требуемые длины кварцевых волокон 120-300 m, поэтому проведение экспериментов по формированию неклассических многофотонных состояний света со сжатыми квантовыми флуктуациями в волокнах из модифицированных висмутом теллуритных стекол является перспективным. При помощи оценочных аналитических формул показано, что участок волокна, на котором сжатие практически перестает расти, связан с влиянием потерь, а участок, на котором сжатие начинает резко ухудшаться - с рамановскими эффектами.

\section{Финансирование работы}

Работа частично поддержана РФФИ (гранты 20-0300874, 19-29-11032), РНФ (грант 20-72-10188 - синтез теллуритного стекла для последующего изготовления микрорезонаторов) и частично поддержана Европейским фондом регионального развития в рамках проекта 1.1.1.2 „Постдокторантура“, Исследовательское финансирование для конкретной цели 1.1.1 „Повышение исследовательского и инновационного потенциала научных учреждений Латвии и возможности привлечения внешнего финансирования, инвестирования в человеческие ресурсы и инфраструктуру“ Оперативной программы „Рост и занятость“" (№ .1.1.1.2/VIAA/4/20/659).

\section{Конфликт интересов}

Авторы заявляют, что у них нет конфликта интересов.

\section{Список литературы}

[1] A.K. Fedorov, A.V. Akimov, J.D. Biamonte, A.V. Kavokin, F.Y. Khalili, E.O. Kiktenko, N.N. Kolachevsky, Y.V. Kurochkin, A.I. Lvovsky, A.N. Rubtsov, G.V. Shlyapnikov, S.S. Straupe, A.V. Ustinov, A.M. Zheltikov. Quantum Sci. Technol., 4 (4), 040501 (2019). DOI: $10.1088 / 2058-9565 / \mathrm{ab} 4472$

[2] F. Gaetano, A. Sascha, F.Y. Khalili, M.V. Chekhova. NPJ Quantum Information, 7 (1), 1-6 (2021). DOI: $10.1038 / \mathrm{s} 41534-021-00407-0$

[3] H.A. Bachor, T.C. Ralph. A guide to experiments in quantum optics (John Wiley \& Sons, Weinheim, 2019). DOI: $10.1002 / 9783527695805$

[4] LIGO Scientific Collaboration and Virgo Collaboration. Phys. Rev. Lett., 116 (6), 061102 (2016). DOI: 10.1103/PhysRevLett.116.061102

[5] G.L. Mansell, T.G. McRae, P.A. Altin, M.J. Yap, R.L. Ward. Phys. Rev. Lett., 120 (20), 203603 (2018). DOI: $10.1103 /$ PhysRevLett.120.203603

[6] U.L. Andersen, T. Gehring, C. Marquardt, G. Leuchs. Physica Scripta., 91 (5), 053001 (2016). DOI: 10.1088/00318949/91/5/053001

[7] A. Canós Valero, D. Kislov, E.A. Gurvitz, H.K. Shamkhi, A.A. Pavlov, D. Redka, S. Yankin, P. Zemánek, A.S. Shalin. Adv. Sci., 7 (11), 1903049 (2020).

DOI: $10.1002 /$ advs.201903049

[8] P.D. Terekhov, A.B. Evlyukhin, D. Redka, V.S. Volkov, A.S. Shalin, A. Karabchevsky. Laser Photonics Rev., 14 (4), 1900331 (2020). DOI: 10.1002/lpor.201900331

[9] H. Barhom, A.A. Machnev, R.E. Noskov, A. Goncharenko, E.A. Gurvitz, A.S. Timin, V.A. Shkoldin, S.V. Koniakhin, O.Yu. Koval, M.V. Zyuzin, A.S. Shalin, I.I. Shishkin, P. Ginzburg. Nano Lett., 19 (10), $7062-7071$ (2019).

DOI: $10.1021 /$ acs.nanolett.9b02540 
[10] O. De Varona, W. Fittkau, P. Booker, T. Theeg, M. Steinke, D. Kracht, J. Neumann, P. Wessels. Optics Express, 25 (21), 24880-24892 (2017). DOI: 10.1364/OE.25.024880

[11] D.P. Kapasi, J. Eichholz, T. McRae, R.L. Ward, B.J.J. Slagmolen, S. Legge, K.S. Hardman, P.A. Altin, D.E. McClelland. Optics Express, 28 (3), 3280-3288 (2020). DOI: $10.1364 / \mathrm{OE} .383685$

[12] Q. Zhang, Y. Hou, X. Wang, W. Song, X. Chen, W. Bin, J. Li, C. Zhao, P. Wang. Optics Letters, 45 (17), 4911-4914 (2020). DOI: $10.1364 /$ OL.402617

[13] K. Bergman, H.A. Haus. Optics Letters, $16(9)$, 663-665 (1991). DOI: 10.1364/OL.16.000663

[14] M. Rosenbluh, R.M. Shelby. Phys. Rev. Lett., 66 (2), 153 (1991). DOI: 10.1103/PhysRevLett.66.153

[15] J.F. Corney, J. Heersink, R. Dong, V. Josse, P.D. Drummond, G. Leuchs, U.L. Andersen. Phys. Rev. A, 78 (2), 023831 (2008). DOI: 10.1103/PhysRevA.78.023831

[16] T.X. Tran, K.N. Cassemiro, C. Söller, K.J. Blow, F. Biancalana. Phys. Rev. A, 84 (1), 013824 (2011). DOI:10.1103/PhysRevA.84.013824

[17] E.A. Anashkina, A.V. Andrianov, J.F. Corney, G. Leuchs. Optics Letters, 45 (19), 5299-5302 (2020). DOI: $10.1364 /$ OL.400326

[18] P. Domachuk, N.A. Wolchover, M. Cronin-Golomb, A. Wang, A.K. George, C.M.B. Cordeiro, J.C. Knight, F.G. Omenetto. Optics Express, 16 (10), 7161-7168 (2008). DOI: $10.1364 /$ OE.16.007161

[19] S. Kedenburg, C. Strutynski, B. Kibler, P. Froidevaux, F. Désévédavy, G. Gadret, J.-C. Jules, T. Steinle, F. Mörz, A. Steinmann, H. Giessen, F. Smektala. J. Opt. Soc. Am. B, 34 (3), 601-607 (2017). DOI: 10.1364/JOSAB.34.000601

[20] L. Zhang, T. Cheng, D. Deng, D. Sega, L. Liu, X. Xue, T. Suzuki; Y. Ohishi. IEEE Photonics Technology Letters, 27 (14), 1547-1549 (2015). DOI: 10.1109/LPT.2015.2429735

[21] M.Yu. Koptev, E.A. Anashkina, A.V. Andrianov, V.V. Dorofeev, A.F. Kosolapov, S.V. Muravyev, A.V. Kim. Optics Letters, 40 (17), 4094-4097 (2015). DOI: 10.1364/OL.40.004094

[22] E.A. Anashkina, A.V. Andrianov, V.V. Dorofeev, A.V. Kim. Applied Optics, 55 (17), 4522-4530 (2016). DOI: $10.1364 / A O .55 .004522$

[23] E.A. Anashkina, V.V. Dorofeev, S.A. Skobelev, A.A. Balakin, S.E. Motorin, A.F. Kosolapov, A.V. Andrianov. Photonics, 7 (3), 51 (2020). DOI: 10.3390/photonics7030051

[24] T. Cheng, L. Zhang, X. Xue, D. Deng, T. Suzuki, Y. Ohishi. Optics Express, 23 (4), 4125-4134 (2015). DOI: $10.1364 / \mathrm{OE} .23 .004125$

[25] P. Wang, L. Chen, X. Zhang, P. Gao, Y. Zhou, W. Zhang, J. Hu, M. Liao, T. Suzuki, Y. Ohishi, W. Gao. Optical and Quantum Electronics, 50 (12), 1-18 (2018). DOI: $10.1007 / \mathrm{s} 11082-018-1680-0$

[26] E.A. Anashkina, A.V. Andrianov. Photonics, 8 (4), 113 (2021). DOI: $10.3390 /$ photonics 8040113

[27] S.A. Skobelev, A.A. Balakin, E.A. Anashkina, A.V. Andrianov, A.G. Litvak. Phys. Rev. A, 104 (3), 033518 (2021). DOI: $10.1103 /$ PhysRevA.104.033518

[28] E.A. Anashkina. Fibers, 8 (5), 30 (2020). DOI: 10.3390/fib8050030

[29] B. Richards, Y. Tsang, D. Binks, J. Lousteau, A. Jha. Optics Letters, 33 (4), 402-404 (2008). DOI: $10.1364 /$ OL.33.000402

[30] B.I. Denker, V.V. Dorofeev, B.I. Galagan, V.V. Koltashev, S.E. Motorin, V.G. Plotnichenko, S.E. Sverchkov. Laser
Physics Letters, 17 (9), 095101 (2020). DOI: 10.1088/1612202X/aba0be

[31] S.V. Muravyev, E.A. Anashkina, A.V. Andrianov, V.V. Dorofeev, S.E. Motorin, M.Y. Koptev, A.V. Kim. Scientific Reports, 8 (1), 1-13 (2018). DOI: 10.1038/s41598-018-34546-w

[32] E.A. Anashkina, V.V. Dorofeev, A.V. Andrianov. Applied Sciences, 11 (12), 5440 (2021).

DOI: doi.org/10.3390/app11125440

[33] E.A. Anashkina, A.V. Andrianov. J. Lightwave Technology, 39 (11), 3568-3574 (2021). DOI: 10.1109/JLT.2021.3064999

[34] R.M. Shelby, M.D. Levenson, P.W. Bayer. Physical review letters, 54 (9), 939 (1985). DOI: 10.1103/PhysRevLett.54.939

[35] A.N. Moiseev, V.V. Dorofeev, A.V. Chilyasov, I.A. Kraev, M.F. Churbanov, T.V. Kotereva, V.G. Pimenov, G.E. Snopatin, A.A. Pushkin, V.V. Gerasimenko, A.F. Kosolapov, V.G. Plotnichenko, E.M. Dianov. Optical Materials, 33 (12), 1858-1861 (2011). DOI: 10.1016/j.optmat.2011.02.042

[36] A.M. Kut'in, A.D. Plekhovich, K.V. Balueva, S.E. Motorin, V.V. Dorofeev. Thermochimica Acta, 673, 192-197 (2019). DOI: $10.1016 /$ j.tca.2019.01.027

[37] M.P. Smayev, V.V. Dorofeev, A.N. Moiseev, A.G. Okhrimchuk. J. of Non-crystalline Solids, 480, 100-106 (2018). DOI: 10.1016/j.jnoncrysol.2017.11.007

[38] G. Qin, M. Liao, T. Suzuki, A. Mori, Y. Ohishi. Optics Letters, 33 (17), 2014-2016 (2008). DOI: 10.1364/OL.33.002014

[39] E.A. Anashkina, A.V. Andrianov, V.V. Dorofeev, V.V. Kim, V.V. Koltashev, G. Leuchs, S.E. Motorin, S.V. Muravyev, A.D. Plekhovich. J. of Non-Crystalline Solids, 525, 119667 (2019). DOI: 10.1016/j.jnoncrysol.2019.119667

[40] A.N. Moiseev, V.V. Dorofeev, A.V. Chilyasov, V.G. Pimenov, T.V. Kotereva, I.A. Kraev, L.A. Ketkova, A.F. Kosolapov, V.G. Plotnichenko, V.V. Koltashev. Inorganic Materials, 47 (6), 665-669 (2011). DOI: 10.1134/S0020168511060161

[41] P.D. Drummond, J.F. Corney. J. Opt. Soc. Am. B, $18(2)$, 139-152 (2001). DOI: 10.1364/JOSAB.18.000139

[42] P.D. Drummond, J.F. Corney. J. Opt. Soc. Am. B, 18(2), 153-161 (2001). DOI: 10.1364/JOSAB.18.000153

[43] A.A. Sorokin, E.A. Anashkina, J.F. Corney, V. Bobrovs, G. Leuchs, A.V. Andrianov. Photonics, 8(6), 226 (2021). DOI: $10.3390 /$ photonics8060226

[44] X. Yan, G. Qin, M. Liao, T. Suzuki, Y. Ohishi. J. Opt. Soc. Am. B, 28 (8), 1831-1836 (2011).

DOI: $10.1364 / J O S A B .28 .001831$ 